\title{
LA INVESTIGACIÓN COMO ÁMBITO DE INSERCIÓN PARA LOS GRADUADOS DE CIENCIAS DE LA EDUCACIÓN: REALIDADES Y PERSPECTIVAS
}

\author{
María Isabel Pozzo (Universidad Nacional de Rosario - \\ Consejo de Investigaciones Científicas y Técnicas)* \\ pozzo@irice-conicet.gov.ar
}

Recibido: 7/08/2012 Aceptado: 7/11/2012

\section{Resumen}

La presente reseña evoca el seminario "La investigación como ámbito de inserción para los graduados de Ciencias de la Educación: realidades y perspectivas" y los temas desarrollados en el mismo. En los acotados márgenes que impone una reseña, se intenta difundir algunas orientaciones muy demandadas por los graduados y aportar a la reflexión sobre la inserción de los mismos en el campo de la investigación.

\section{Palabras clave \\ Investigación - Educación - Graduados.}

El título de esta reseña alude al seminario homónimo organizado por la Escuela de Ciencias de la Educación de la Facultad de Humanidades y Artes de la Universidad Nacional de Rosario (UNR), la Asociación de Graduados en Ciencias de la Educación de Rosario (AGCER) y el Instituto Rosario de Investigaciones en Ciencias de la Educación (IRICE) del Consejo Nacional de

* Doctora en Ciencias de la Educación por la Universidad Nacional de Rosario. Magister en Formación de Profesores de Español como Lengua Extranjera por la Universitat de Barcelona, España. Profesora y Licenciada en Ciencias de la Educación. Universidad Nacional de Rosario. Investigadora del CONICET, Coordinadora del Área de Estudios Interculturales del Instituto Rosario de Investigaciones en Ciencias de la Educación (IRICECONICET). 
Investigaciones Científicas y Tecnológicas (CONICET). El mismo tuvo lugar el 21 de noviembre de 2011 y estuvo a cargo de quien suscribe.

El seminario se llevó a cabo con la intención de dar respuesta a la demanda de los graduados de esta carrera de intensificar su formación en investigación. Esta necesidad fue expresada en la Jornada: "Los caminos del cientista. Encuentro entre graduados y estudiantes de Ciencias de la Educación”, organizada por dicha Escuela y AGCER el 26 de Octubre de 2011. La Jornada proponía reflexionar sobre el paso del ámbito universitario al laboral. Tras el panel de apertura a cargo de las autoridades -Dra. María Silvia Serra, directora de la Escuela de Ciencias de la Educación y Prof. Laura Giorgiani, Presidente de AGCER-, los asistentes deliberaron en tres comisiones temáticas: docencia; investigación y asesoramiento y otros espacios. El evento concluyó con una puesta en común de las reflexiones surgidas en cada espacio y el consenso generalizado sobre la necesidad de continuar el desarrollo de las temáticas de las comisiones.

En este contexto, el seminario "La investigación como ámbito de inserción para los graduados de Ciencias de la Educación: realidades y perspectivas" persiguió tres propósitos: presentar una descripción de la inserción actual de los graduados de Ciencias de la Educación de la Universidad Nacional de Rosario en el ámbito de la investigación (las realidades, según el título del evento); informar acerca de los principales mecanismos e instituciones de dicho ámbito (las perspectivas) y de este modo promover la inserción de los graduados en él.

Para el primer propósito, el indicador más contundente es la comparación del número de Licenciados y Profesores, considerando que la Licenciatura constituye la especialización de la carrera en la investigación. El análisis de las cohortes permite apreciar que en todos los años, excepto en 1996, la mayoría de los graduados son profesores. De un total de 812 graduados desde la primera promoción hasta la fecha del seminario, 709 son profesores y 103 licenciados. Esta brecha se advierte claramente en el cuadro $\mathrm{N}^{0} 1$ elaborado para el evento a partir de los listados de Sección Alumnado de la Facultad.

Cuadro N¹: Profesores y Licenciados en Ciencias de la Educación (UNR) desde la primera promoción (1989) a noviembre de 2011

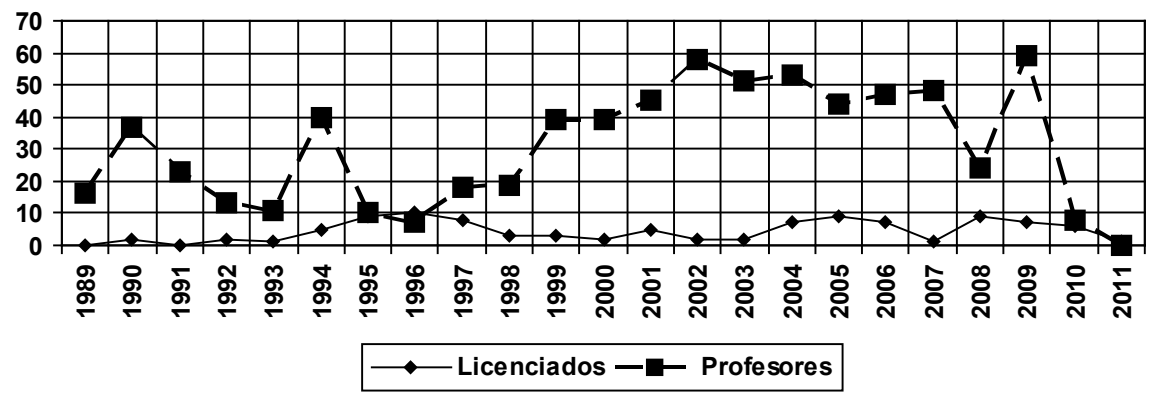


Las causas de ello podrían hipotetizarse en:

- $\quad$ el imaginario de que el título de profesor provee más oportunidades de inserción que el de licenciado.

- la sensación de desamparo que genera en los tesistas un recorrido individual y con plazos menos circunscriptos.

Más allá del acierto o desacierto de estas creencias, las cifras presentadas justifican la necesidad de transmitir saberes vinculados al desempeño en el campo de la investigación, segundo objetivo del seminario. Entre ellos, se señalaron espacios de vinculación capaces de suplir el vacío que deviene al cabo del cursado de la carrera. Algunas instancias recomendadas para buscar la inserción son la adscripción a una cátedra -sea de la institución de origen u otra-, la incorporación a un Centro de Estudios (que en la Facultad de Humanidades y Artes suman cincuenta) y/o la participación en un equipo de investigación. Entre las titulaciones se difundió la Licenciatura y los posgrados en sus distintos alcances.

El itinerario prosiguió con las principales instituciones subsidiantes de investigaciones: la Agencia Nacional de Promoción Científica y Tecnológica, el Consejo Nacional de Investigaciones Científicas y Técnicas (CONICET) y a nivel local, el Consejo de Investigación de la UNR (CIUNR). Especial énfasis se puso en el CONICET, que cuenta con una convocatoria anual permanente de becas que en los últimos años suman el $40 \%$ de las que se otorgan a nivel nacional. Desde la consolidación democrática, las Ciencias Sociales y Humanidades han ganado un espacio mayor, liderando en el 2011 con un 32\% -a la par de las Ciencias Biológicas y de la Salud- según se desprende del portal del propio Consejo. No obstante, la comisión de Filosofía, Psicología y Cs. de la Educación abarca el $19 \%$ del total de becas y la restricción que se produce en el paso de becario a investigador es notoria.

Tras este encuadre nacional, la exposición se focalizó en la carrera de Ciencias de la Educación (UNR). Datos de primera mano (cuadro $\mathrm{N}^{\circ} 2$ ) dejan ver que la investigación constituye un ámbito con poca presencia de los cientistas de la educación. No obstante, la desestimación del mismo por parte de los graduados no contribuye a que dicha situación se modifique. Por otra parte, el desconocimiento del modus operandi de estas instituciones se convierte en un aliado que confina a los cientistas a los roles más tradicionales.

La activa participación de los asistentes permite concluir que eventos como este seminario y la jornada que le antecedió, contribuyen a relativizar los mitos y a acercar las realidades para que la investigación educativa prolifere como lugar de inserción del cientista de la educación.

La sinergia institucional constituyó un aspecto destacable, ya que -si bien temáticamente afines- son nulos los antecedentes conjuntos de esta trilogía. Dado el potencial de las tres instituciones involucradas, celebramos el trabajo 
mancomunado y anhelamos que los eventos reseñados sean el inicio de nuevas actividades compartidas.

Cuadro $N^{\circ}$ 2: Becarios e investigadores de CIUNR y CONICET en la planta docente de la carrera de Ciencias de la Educación (UNR) en noviembre de 2011

\begin{tabular}{|c|c|c|c|}
\hline & Total & $\begin{array}{l}\text { Carrera } \\
\text { de grado cursada }\end{array}$ & $\begin{array}{l}\text { Espacio curricular } \\
\text { en el que se } \\
\text { desempeña }\end{array}$ \\
\hline Becarios CONICET & 4 & $\begin{array}{l}3 \text { Cs. de la Educ. } \\
\text { (UNR) } \\
1 \mathrm{Cs} \text {. de la Educ } \\
\text { (UNQ) }\end{array}$ & $\begin{array}{l}2 \text { en Núcleo Socio- } \\
\text { educativo } \\
1 \text { en Área del } \\
\text { Sujeto } \\
1 \text { en Área de las } \\
\text { Instituciones }\end{array}$ \\
\hline $\begin{array}{l}\text { Investigadores } \\
\text { CONICET }\end{array}$ & 1 & Cs. de la Educ. (UNR) & Trabajo de Campo \\
\hline Becarios CIUNR & 0 & - & - \\
\hline $\begin{array}{l}\text { Investigadores } \\
\text { CIUNR }\end{array}$ & 2 & $\begin{array}{l}\text { Psicología (UNR) } \\
\text { Antropología (UNR) }\end{array}$ & $\begin{array}{l}\text { Área del Sujeto } \\
\text { Problemática } \\
\text { Antropológica }\end{array}$ \\
\hline
\end{tabular}

